\title{
Precise Asymptotics for Complete Integral Convergence under Sublinear Expectations
}

\author{
Qunying Wu 10 \\ College of Science, Guilin University of Technology, Guilin 541004, China \\ Correspondence should be addressed to Qunying Wu; wqy666@glut.edu.cn
}

Received 21 December 2019; Accepted 28 February 2020; Published 5 May 2020

Academic Editor: Chaudry M. Khalique

Copyright ( $\odot 2020$ Qunying Wu. This is an open access article distributed under the Creative Commons Attribution License, which permits unrestricted use, distribution, and reproduction in any medium, provided the original work is properly cited.

\begin{abstract}
The aim of this paper is to study and establish the precise asymptotics for complete integral convergence theorems under a sublinear expectation space. As applications, the precise asymptotics for $p(0 \leq p \leq 2)$ order complete integral convergence theorems have been generalized to the sublinear expectation space context. We extend some precise asymptotics for complete moment convergence theorems from the traditional probability space to the sublinear expectation space. Our results generalize corresponding results obtained by Liu and Lin (2006). There is no report on the precise asymptotics under sublinear expectation, and we provide the method to study this subject.
\end{abstract}

\section{Introduction}

The sublinear expectation space has advantages of modelling the uncertainty of probability and distribution. Therefore, limit theorems for sublinear expectations have raised a large number of issues of interest recently. Limit theorems are important research topics in probability and statistics. They were widely used in finance and other fields. Classical limit theorems only hold in the case of model certainty. However, in practice, such model certainty assumption is not realistic in many areas of applications because the uncertainty phenomena cannot be modeled using model certainty. Motivated by modelling uncertainty in practice, Peng [1] introduced a new notion of sublinear expectation. As an alternative to the traditional probability/expectation, capacity/sublinear expectation has been studied in many fields such as statistics, finance, economics, and measures of risk (see Denis and Martini [2]; Gilboa [3]; Marinacci [4]; Peng $[1,5-7]$, etc.). The general framework of the sublinear expectation in a general function space was introduced by Peng $[1,7,8]$, and sublinear expectation is a natural extension of the classical linear expectation.

Because the sublinear expectation provides a very flexible framework to model sublinear probability problems, the limit theorems of the sublinear expectation have received more and more attention and research recently. A series of useful results have been established. Peng $[1,7,8]$ constructed the basic framework, basic properties, and central limit theorem under sublinear expectations, Zhang [9-11] established the exponential inequalities, Rosenthal's inequalities, strong law of large numbers, and law of iterated logarithm, $\mathrm{Hu}$ [12], Chen [13], and $\mathrm{Wu}$ and Jiang [14] studied strong law of large numbers, Wu et al. [15] studied the asymptotic approximation of inverse moment, Xi et al. [16] and Lin and Feng [17] studied complete convergence, and so on. In general, extending the limit properties of conventional probability space to the cases of sublinear expectation is highly desirable and of considerably significance in the theory and application. Because sublinear expectation and capacity is not additive, many powerful tools and common methods for linear expectations and probabilities are no longer valid, so that the study of the limit theorems under sublinear expectation becomes much more complex and difficult.

Since the concept of complete convergence of a sequence of random variables was introduced by $\mathrm{Hsu}$ and Robbins [18], there have been extensions in several directions. One of them is to discuss the precise rate, which is more exact than complete convergence. Precise asymptotics for complete convergence and complete moment convergence is one of 
the most important problems in probability theory. Many related results have been obtained in the probabilistic space. Their recent results can be found in the studies of Heyde [19]; Liu and Lin [20]; Zhao [21]; Li [22]; Zhou [23]; Gut and Steinebach [24]; He and Xie [25]; Wang et al. [26-29]; Spaataru [30]; and Kong and Dai [31]. However, in sublinear expectations, due to the uncertainty of expectation and capacity, the precise asymptotics is essentially different from the ordinary probability space. The study of precise asymptotics of complete convergence and complete integral convergence for sublinear expectations is much more complex and difficult. The precise asymptotics theorems under sublinear expectation have not been reported. The purpose of this paper is to establish the precise asymptotics theorems for $p(0 \leq p \leq 2)$ order complete integral convergence for independent and identically distributed random variables under sublinear expectation. As a result, the corresponding results obtained by Liu and Lin [20] have been generalized to the sublinear expectation space context.

In the next section, we summarize some basic notations and concepts and related properties under the sublinear expectations.

\section{Preliminaries}

We use the framework and notations of Peng [8]. Let $(\Omega, \mathscr{F})$ be a given measurable space, and let $\mathscr{H}$ be a linear space of real functions defined on $(\Omega, \mathscr{F})$ such that if $X_{1}, \ldots$, $X_{n} \in \mathscr{H}$, then $\varphi\left(X_{1}, \ldots, X_{n}\right) \in \mathscr{H}$ for each $\varphi \in C_{l, \text { Lip }}\left(\mathbb{R}_{n}\right)$, where $C_{l, \text { Lip }}\left(\mathbb{R}_{n}\right)$ denotes the linear space of (local Lipschitz) functions $\varphi$ satisfying

$$
|\varphi(\mathbf{x})-\varphi(\mathbf{y})| \leq c\left(1+|\mathbf{x}|^{m}+|\mathbf{y}|^{m}\right)|\mathbf{x}-\mathbf{y}|, \quad \forall \mathbf{x}, \mathbf{y} \in \mathbb{R}_{n},
$$

for some $c>0, m \in \mathbb{N}$, depending on $\varphi$. $\mathscr{H}$ is considered as a space of "random variables." In this case, we denote $X \in \mathscr{H}$.

Definition 1. A sublinear expectation $\widehat{\mathbb{E}}$ on $\mathscr{H}$ is a function $\widehat{\mathbb{E}}: \mathscr{H} \longrightarrow[-\infty, \infty]$ satisfying the following properties: for all $X, Y \in \mathscr{H}$,

(a) Monotonicity: If $X \geq Y$, then $\widehat{\mathbb{E}} X \geq \widehat{\mathbb{E}} Y$

(b) Constant preserving: $\widehat{\mathbb{E}} c=c$

(c) Subadditivity: $\widehat{\mathbb{E}}(X+Y) \leq \widehat{\mathbb{E}} X+\widehat{\mathbb{E}} Y \quad$ whenever $\widehat{\mathbb{E}} X+\widehat{\mathbb{E}} Y$ is not of the form $+\infty-\infty$ or $-\infty+\infty$

(d) Positive homogeneity: $\widehat{\mathbb{E}}(\lambda X)=\lambda \widehat{\mathbb{E}} X, \lambda \geq 0$ space.

The triple $(\Omega, \mathscr{H}, \widehat{\mathbb{E}})$ is called a sublinear expectation

Given a sublinear expectation $\widehat{\mathbb{E}}$, let us denote the conjugate expectation $\widehat{\mathcal{E}}$ of $\widehat{\mathbb{E}}$ by

$$
\widehat{\mathcal{E}} X:=-\widehat{\mathbb{E}}(-X), \quad \forall X \in \mathscr{H} .
$$

From the definition, it is easily shown that for all $X, Y \in \mathscr{H}$,

$$
\begin{gathered}
\hat{\varepsilon} X \leq \hat{\mathbb{E}} X, \\
\hat{\mathbb{E}}(X+c)=\hat{\mathbb{E}} X+c, \\
|\hat{\mathbb{E}}(X-Y)| \leq \hat{\mathbb{E}}|X-Y|, \\
\hat{\mathbb{E}}(X-Y) \geq \hat{\mathbb{E}} X-\hat{\mathbb{E}} Y .
\end{gathered}
$$

If $\widehat{\mathbb{E}} Y=\widehat{\mathcal{\varepsilon}} Y$, then $\widehat{\mathbb{E}}(X+a Y)=\widehat{\mathbb{E}} X+a \widehat{\mathbb{E}} Y$ for any $a \in \mathbb{R}$. Next, we consider the capacities corresponding to the sublinear expectations. Let $\mathscr{G} \subset \mathscr{F}$. A function $V: \mathscr{G} \longrightarrow[0,1]$ is called a capacity if

$$
\begin{aligned}
& V(\varnothing)=0, \\
& V(\Omega)=1, \\
& V(A) \leq V(B), \quad \text { for } \forall A \subseteq B, A, B \in \mathscr{G} .
\end{aligned}
$$

It is called to be subadditive if $V(A \cup B) \leq V(A)+V(B)$ for all $A, B \in \mathscr{G}$ with $A \cup B \in \mathscr{G}$. In the sublinear space $(\Omega, \mathscr{H}, \widehat{\mathbb{E}})$, we denote a pair $(\mathbb{V}, \nu)$ of capacities by

$\mathbb{V}(A):=\inf \{\widehat{\mathbb{E}} \xi ; I(A) \leq \xi, \xi \in \mathscr{H}\}, \nu(A):=1-\mathbb{V}\left(A^{c}\right), \forall A \in \mathscr{F}$,

where $A^{c}$ is the complement set of $A$. By definition of $\mathbb{V}$ and $\nu$, it is obvious that $\mathbb{V}$ is subadditive, and

$$
\begin{gathered}
\nu(A) \leq \mathbb{V}(A), \quad \forall A \in \mathscr{F} ; \\
\mathbb{V}(A)=\widehat{\mathbb{E}}(I(A)), \nu(A)=\widehat{\mathcal{\varepsilon}}(I(A)), \quad \text { if } I(A) \in \mathscr{H}, \\
\widehat{\mathbb{E}} f \leq \mathbb{V}(A) \leq \widehat{\mathbb{E}} g, \widehat{\varepsilon} f \leq \nu(A) \leq \widehat{\mathcal{\varepsilon}} g, \text { if } f \leq I(A) \leq g, f, g \in \mathscr{H} .
\end{gathered}
$$

This implies Markov inequality: $\forall X \in \mathscr{H}$,

$$
\mathbb{V}(|X| \geq x) \leq \frac{\widehat{\mathbb{E}}\left(|X|^{p}\right)}{x^{p}}, \quad \forall x>0, p>0,
$$

from $I(|X| \geq x) \leq|X|^{p} / x^{p} \in \mathscr{H}$. By Lemma 4.1 in Zhang [10]; we have Holder inequality: $\forall X, Y \in \mathscr{H}, p, q>1$ satisfying $p^{-1}+q^{-1}=1$,

$$
\widehat{\mathbb{E}}(|X Y|) \leq\left(\widehat{\mathbb{E}}\left(|X|^{p}\right)\right)^{1 / p}\left(\widehat{\mathbb{E}}\left(|Y|^{q}\right)\right)^{1 / q} .
$$

And particularly, we have Jensen inequality: $\forall X \in \mathscr{H}$,

$$
\left(\widehat{\mathbb{E}}\left(|X|^{r}\right)\right)^{1 / r} \leq\left(\widehat{\mathbb{E}}\left(|X|^{s}\right)\right)^{1 / s}, \quad \text { for } 0<r \leq s .
$$

Also, we define the Choquet integrals/expectations $\left(C_{\mathbb{V}}, C_{\nu}\right)$ by

$$
C_{V}(X):=\int_{0}^{\infty} V(X>x) \mathrm{d} x+\int_{-\infty}^{0}(V(X>x)-1) \mathrm{d} x
$$

with $V$ being replaced by $\mathbb{V}$ and $\nu$, respectively.

Definition 2 (Peng [1] and Zhang [9]).

(i) Identical distribution: let $\mathbf{X}_{1}$ and $\mathbf{X}_{2}$ be two $n$-dimensional random vectors defined, respectively, in sublinear expectation spaces $\left(\Omega_{1}, \mathscr{H}_{1}, \widehat{\mathbb{E}}_{1}\right)$ and $\left(\Omega_{2}, \mathscr{H}_{2}, \widehat{\mathbb{E}}_{2}\right)$. They are called identically distributed if 


$$
\widehat{\mathbb{E}}_{1}\left(\varphi\left(\mathbf{X}_{1}\right)\right)=\widehat{\mathbb{E}}_{2}\left(\varphi\left(\mathbf{X}_{2}\right)\right), \quad \forall \varphi \in C_{l, \text { Lip }}\left(\mathbb{R}_{n}\right),
$$

whenever the subexpectations are finite. A sequence $\left\{X_{n} ; n \geq 1\right\}$ of random variables is said to be identically distributed if for each $i \geq 1, X_{i}$ and $X_{1}$ are identically distributed.

(ii) Independence: in a sublinear expectation space $(\Omega, \mathscr{H}, \widehat{\mathbb{E}})$, a random vector $\mathbf{Y}=\left(Y_{1}, \ldots, Y_{n}\right)$, $Y_{i} \in \mathscr{H}$, is said to be independent of another random vector $\mathbf{X}=\left(X_{1}, \ldots, X_{m}\right), \quad X_{i} \in \mathscr{H}$, under $\widehat{\mathbb{E}}$ if for each test function $\varphi \in C_{l, L i p}\left(\mathbb{R}_{m} \times \mathbb{R}_{n}\right)$, we have $\widehat{\mathbb{E}}(\varphi(\mathbf{X}, \mathbf{Y}))=\widehat{\mathbb{E}}\left[\left.\widehat{\mathbb{E}}(\varphi(\mathbf{x}, \mathbf{Y}))\right|_{\mathbf{X}=\mathbf{X}}\right]$, whenever $\bar{\varphi}(\mathbf{x}):=\widehat{\mathbb{E}}(|\varphi(\mathbf{x}, \mathbf{Y})|)<\infty$ for all $\mathbf{x}$ and $\widehat{\mathbb{E}}(|\bar{\varphi}(\mathbf{X})|)<\infty$. (iii) Independent random variables: a sequence of random variables $\left\{X_{n} ; n \geq 1\right\}$ is said to be independent, if $X_{i+1}$ is independent of $\left(X_{1}, \ldots, X_{i}\right)$ for each $i \geq 1$.

In the following, let $\left\{X_{n} ; n \geq 1\right\}$ be a sequence of random variables in $(\Omega, \mathscr{H}, \widehat{\mathbb{E}})$ and $S_{n}=\sum_{i=1}^{n} X_{i}$. The symbol $c$ stands for a generic positive constant which may differ from one place to another. Let $a_{x} \sim b_{x}$ denote $\lim _{x \longrightarrow \infty} a_{x} / b_{x}=1$, $a_{x} \ll b_{x}$ denote that there exists a constant $c>0$ such that $a_{x} \leq c b_{x}$ for sufficiently large $x$, and $I(\cdot)$ denote an indicator function.

To prove our results, we need the following four lemmas.

Lemma 1 (Theorem 3.1 in Zhang [10]). Let $\left\{X_{k} ; k \geq 1\right\}$ be a sequence of independent random variables in $(\Omega, \mathscr{H}, \widehat{\mathbb{E}})$ with $\widehat{\mathbb{E}} X_{k} \leq 0$. Then,

(i) For any $x, y>0$,

$$
\mathbb{V}\left(S_{n} \geq x\right) \leq \mathbb{V}\left(\max _{1 \leq k \leq n} X_{k}>y\right)+\exp \left(-\frac{x^{2}}{2\left(x y+B_{n}\right)}\left\{1+\frac{2}{3} \ln \left(1+\frac{x y}{B_{n}}\right)\right\}\right)
$$

(ii) If $\widehat{\mathbb{E}} X_{k}=\widehat{\mathbb{E}}\left(-X_{k}\right)=0$, then

$$
\mathbb{V}\left(\left|S_{n}\right| \geq x\right) \leq c \frac{B_{n}}{x^{2}}
$$

where $B_{n}=\sum_{k=1}^{n} \widehat{\mathbb{E}} X_{k}^{2}$.

Lemma 2. For any $X \in \mathscr{H}$, we have

$$
\begin{gathered}
C_{\mathbb{V}}\left(X^{2}\right)<\infty \Longleftrightarrow \int_{1}^{\infty} x \mathbb{V}(|X|>x) \mathrm{d} x<\infty, \\
C_{\mathbb{V}}\left(X^{2} \ln |X|\right)<\infty \Longleftrightarrow \int_{3}^{\infty} x \ln x \mathbb{V}(|X|>x) \mathrm{d} x<\infty
\end{gathered}
$$

Proof. We only prove (16). Let $f(x):=x^{2} \ln x$ and $f^{-1}(\cdot)$ denote the inverse function of $f(\cdot)$. Then, (16) follows from the following three equations:

$$
\begin{gathered}
\int_{f(3)}^{\infty} \mathbb{V}\left(X^{2} \ln |X|>x\right) \mathrm{d} x \leq C_{\mathbb{V}}\left(X^{2} \ln |X|\right) \leq f(3)+\int_{f(3)}^{\infty} \mathbb{V}\left(X^{2} \ln |X|>x\right) \mathrm{d} x, \\
\int_{f(3)}^{\infty} \mathbb{V}\left(X^{2} \ln |X|>x\right) \mathrm{d} x=\int_{f(3)}^{\infty} \mathbb{V}\left(|X|>f^{-1}(x)\right) \mathrm{d} x=\int_{3}^{\infty}(2 y \ln y+y) \mathbb{V}(|X|>y) \mathrm{d} y\left(\operatorname{let} f^{-1}(x)=y\right), \\
2 y \ln y \leq 2 y \ln y+y \leq 3 y \ln y, \\
\text { for } y \geq 3
\end{gathered}
$$

Therefore, (16) holds.

Here, we give the notations of G-normal distribution which is introduced by Peng [7].

Definition 3 (G-normal random variable). For $0 \leq \underline{\sigma}^{2} \leq \bar{\sigma}^{2}<\infty$, a random variable $\xi$ in a sublinear expectation space $(\Omega, \mathscr{H}, \widehat{\mathbb{E}})$ is called a normal $\mathcal{N}\left(0,\left[\underline{\sigma}^{2}, \bar{\sigma}^{2}\right]\right)$ distributed random variable (write $\xi \sim \mathcal{N}\left(0,\left[\underline{\sigma}^{2}, \bar{\sigma}^{2}\right]\right)$ under $\widehat{\mathbb{E}})$; if for any $\varphi \in C_{l, L i p}(\mathbb{R})$, the function $u(x, t)=\widehat{\mathbb{E}}(\varphi(x+\sqrt{t} \xi))(x \in \mathbb{R}, t \geq 0)$ is the unique viscosity solution of the following heat equation:

$$
\begin{aligned}
\partial_{t} u-G\left(\partial_{x x}^{2} u\right) & =0, \\
u(0, x) & =\varphi(x),
\end{aligned}
$$

where $G(\alpha)=\left(\bar{\sigma}^{2} \alpha^{+}-\underline{\sigma}^{2} \alpha^{-}\right) / 2$.

Lemma 3 (Theorem 3.3 and Remark 3.4 in Peng [7] (CLT)). Suppose that $\left\{X_{n} ; n \geq 1\right\}$ is a sequence of independent and identically distributed random variables with $\widehat{\mathbb{E}}\left(X_{1}\right)=\widehat{\mathbb{E}}\left(-X_{1}\right)=0$. Write $\bar{\sigma}^{2}=\widehat{\mathbb{E}}\left(X_{1}^{2}\right)$ and $\underline{\sigma}^{2}=\widehat{\mathcal{\varepsilon}}\left(X_{1}^{2}\right)$. Then, for any continuous function $\varphi$ satisfying $|\varphi(x)| \leq c(1+|x|)$, 


$$
\lim _{n \rightarrow \infty} \widehat{\mathbb{E}}\left(\varphi\left(\frac{S_{n}}{\sqrt{n}}\right)\right)=\widehat{\mathbb{E}}(\varphi(\xi)),
$$

where $\xi \sim \mathcal{N}\left(0,\left[\underline{\sigma}^{2}, \bar{\sigma}^{2}\right]\right)$ under $\widehat{\mathbb{E}}$.

In particular, if $\bar{\sigma}=\underline{\sigma}$, then Lemma 3 becomes a classical central limit theorem.

Remark 1. For any $x>0$, by $\widehat{\mathbb{E}}\left(x X_{1}\right)^{2}=x^{2} \bar{\sigma}^{2}$, $\widehat{\mathcal{E}}\left(x X_{1}\right)^{2}=x^{2} \underline{\sigma}^{2}$, and $x \xi \sim \mathcal{N}\left(0,\left[x^{2} \underline{\sigma}^{2}, x^{2} \bar{\sigma}^{2}\right]\right)$ under $\widehat{\mathbb{E}},(19)$ becomes

$$
\lim _{n \longrightarrow \infty} \widehat{\mathbb{E}}\left(\varphi\left(\frac{x S_{n}}{\sqrt{n}}\right)\right)=\widehat{\mathbb{E}}(\varphi(x \xi)) .
$$

Lemma 4 (Lemma 3 in Chen and $\mathrm{Hu}$ [32]). Suppose that $\xi \sim \mathcal{N}\left(0,\left[\underline{\sigma}^{2}, \bar{\sigma}^{2}\right]\right)$ under $\widehat{\mathbb{E}}$. Let $P$ be a probability measure and $\varphi$ be a bounded continuous function on $\mathbb{R}$. If $\left\{B_{t}\right\}_{t \geq 0}$ is a Brownian motion under $P$, then

$$
\widehat{\mathbb{E}}(\varphi(\xi))=\sup _{\theta \in \Theta} E_{P}\left[\varphi\left(\int_{0}^{1} \theta_{s} \mathrm{~d} B_{s}\right)\right],
$$

where

$$
\begin{aligned}
\Theta & =\left\{\theta ; \theta_{t} i \text { i } \mathscr{F}_{t}-\text { adapted process such that } \underline{\sigma} \leq \theta_{t} \leq \bar{\sigma}\right\}, \\
\mathscr{F}_{t} & =\sigma\left\{B_{s} ; 0 \leq s \leq t\right\} \vee \mathcal{N}, \mathcal{N} \text { is the collection of } P-\text { null subsets. }
\end{aligned}
$$

From Peng [8], if $\xi \sim \mathcal{N}\left(0,\left[\underline{\sigma}^{2}, \bar{\sigma}^{2}\right]\right)$ under $\widehat{\mathbb{E}}$, then for each convex function $\varphi$,

$$
\widehat{\mathbb{E}}(\varphi(\xi))=\frac{1}{\sqrt{2 \pi}} \int_{-\infty}^{\infty} \varphi(\bar{\sigma} x) \mathrm{e}^{-x^{2} / 2} \mathrm{~d} x,
$$

but if $\varphi$ is a concave function, the above $\bar{\sigma}$ must be replaced by $\underline{\sigma}$. If $\sigma=\bar{\sigma}=\underline{\sigma}$, then $\mathcal{N}\left(0,\left[\underline{\sigma}^{2}, \bar{\sigma}^{2}\right]\right)=\mathcal{N}\left(0, \sigma^{2}\right)$ which is a classical normal distribution.

In particular, notice that $\varphi(x)=|x|^{p}, p \geq 1$, is a convex function; taking $\varphi(x)=|x|^{p}, p \geq 1$, in (23), we get

$$
\widehat{\mathbb{E}}\left(|\xi|^{p}\right)=\frac{2 \bar{\sigma}^{p}}{\sqrt{2 \pi}} \int_{0}^{\infty} x^{p} \mathrm{e}^{-x^{2} / 2} \mathrm{~d} x<\infty
$$

(24) implies that

$$
\begin{aligned}
C_{\mathbb{V}}\left(|\xi|^{p}\right)= & \int_{0}^{\infty} \mathbb{V}\left(|\xi|^{p}>x\right) \mathrm{d} x \leq 1 \\
& +\int_{1}^{\infty} \frac{\widehat{\mathbb{E}}\left(|\xi|^{2 p}\right)}{x^{2}} \mathrm{~d} x<\infty, \quad \text { for any } p \geq \frac{1}{2} .
\end{aligned}
$$

Definition 4. A sublinear expectation $\widehat{\mathbb{E}}$ is called to be continuous if it satisfies

Continuity from below: $\widehat{\mathbb{E}}\left(X_{n}\right) \uparrow \widehat{\mathbb{E}}(X)$ if $0 \leq X_{n} \uparrow X$, where $X_{n}, X \in \mathscr{H}$

Continuity from above: $\widehat{\mathbb{E}}\left(X_{n}\right) \downarrow \widehat{\mathbb{E}}(X)$ if $0 \leq X_{n} \downarrow X$, where $X_{n}, X \in \mathscr{H}$
Lemma 5. Suppose that the conditions of Lemma 3 hold and $\widehat{\mathbb{E}}$ is continuous, set $\Delta_{n}(x):=\mathbb{V}\left(\left(\left|S_{n}\right| / \sqrt{n}\right) \geq x\right)-\mathbb{V}(|\xi| \geq x)$, here and later, $\xi \sim \mathcal{N}\left(0,\left[\underline{\sigma}^{2}, \bar{\sigma}^{2}\right]\right)$ under $\widehat{\mathbb{E}}$; then,

$$
\Delta_{n}:=\sup _{x \geq 0}\left|\Delta_{n}(x)\right| \longrightarrow 0, \quad \text { as } n \longrightarrow \infty \text {. }
$$

Remark 2. Lemma 5 is a powerful tool for studying the uniform convergence of the central limit theorem under sublinear expectations, which plays a key role in proving the theorems in this paper.

Proof of Lemma 5. If $\bar{\sigma}=\underline{\sigma}$, then Lemma 3 is a classical central limit theorem. In the classical probability, (26) follows from the central limit theorem and an important fact that $P(|\xi| \geq x)$ is a continuous function of $x$. Therefore, we only need to prove the situation $\underline{\sigma}<\bar{\sigma}$.

Obviously, $\Delta_{n}(0)=1-1=0$; thus, $\Delta_{n}=\sup _{x>0}\left|\Delta_{n}(x)\right|$.

For $0<\mu<1$, let $\varphi(x)$ be a Lipschitz even function and nondecreasing for $x \geq 0$ such that $0 \leq \varphi(x) \leq 1$, for all $x$ and $\varphi(x)=0$ if $|x| \leq \mu$ and $\varphi(x)=1$ if $|x|>1$. Then,

$$
I(|x| \geq 1) \leq \varphi(x) \leq I(|x| \geq \mu) .
$$

This combines equation (7), for $x>0$,

$$
\begin{aligned}
\Delta_{n}(x) & \leq \hat{\mathbb{E}}\left[\varphi\left(\frac{S_{n}}{\sqrt{n} x}\right)\right]-\hat{\mathbb{E}}\left[\varphi\left(\frac{\mu \xi}{x}\right)\right] \\
& =\hat{\mathbb{E}}\left[\varphi\left(\frac{S_{n}}{\sqrt{n} x}\right)\right]-\hat{\mathbb{E}}\left[\varphi\left(\frac{\xi}{x}\right)\right]+\hat{\mathbb{E}}\left[\varphi\left(\frac{\xi}{x}\right)\right]-\hat{\mathbb{E}}\left[\varphi\left(\frac{\mu \xi}{x}\right)\right] \\
& :=\Delta_{n 1}(x)+\Delta_{2}(x) \leq \sup _{x>0}\left|\Delta_{n 1}(x)\right|+\sup _{x>0} \Delta_{2}(x),
\end{aligned}
$$

where $\quad \Delta_{n 1}(x)=\widehat{\mathbb{E}}\left[\varphi\left(S_{n} / \sqrt{n} x\right)\right]-\widehat{\mathbb{E}}[\varphi(\xi / x)] \quad$ and $\Delta_{2}(x)=\widehat{\mathbb{E}}[\varphi(\xi / x)]-\widehat{\mathbb{E}}[\varphi(\mu \xi / x)] \geq 0$.

On the other hand,

$$
\begin{aligned}
\Delta_{n}(x) & \geq \hat{\mathbb{E}}\left[\varphi\left(\frac{\mu S_{n}}{\sqrt{n} x}\right)\right]-\hat{\mathbb{E}}\left[\varphi\left(\frac{\xi}{x}\right)\right] \\
& =\hat{\mathbb{E}}\left[\varphi\left(\frac{\mu S_{n}}{\sqrt{n} x}\right)\right]-\hat{\mathbb{E}}\left[\varphi\left(\frac{\mu \xi}{x}\right)\right]+\hat{\mathbb{E}}\left[\varphi\left(\frac{\mu \xi}{x}\right)\right]-\hat{\mathbb{E}}\left[\varphi\left(\frac{\xi}{x}\right)\right] \\
& =\Delta_{n 1}\left(\frac{x}{\mu}\right)-\Delta_{2}(x) \geq-\sup _{x>0}\left|\Delta_{n 1}(x)\right|-\sup _{x>0} \Delta_{2}(x) .
\end{aligned}
$$

Thus,

$$
\Delta_{n}=\sup _{x>0}\left|\Delta_{n}(x)\right| \leq \sup _{x>0}\left|\Delta_{n 1}(x)\right|+\sup _{x>0} \Delta_{2}(x) .
$$
that

Therefore, in order to prove that (26), it suffices to show

$$
\begin{array}{r}
\lim _{n \longrightarrow \infty} \sup _{x>0}\left|\Delta_{n 1}(x)\right|=0, \\
\lim _{\mu \longrightarrow 1^{-}} \sup _{x>0} \Delta_{2}(x)=0 .
\end{array}
$$




$$
\begin{aligned}
& \text { Write } \\
& \begin{cases}\hat{\mathbb{E}}(\varphi(\xi / x)) & F_{n}(x)=\hat{\mathbb{E}}\left(\varphi\left(S_{n} / \sqrt{n} x\right)\right) \\
1 & x=0\end{cases}
\end{aligned}
$$

Obviously, $0 \leq F_{n}(x)$ and $F(x) \leq 1 ; F_{n}(x)$ and $F(x)$ are nonincreasing functions on $[0,+\infty)$. Thus, for any $x_{0}>0$,

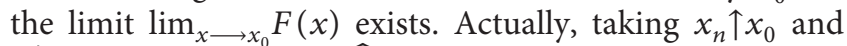
$x_{n}^{\prime} \downarrow x_{0}$, by continuity of $\widehat{\mathbb{E}}$, we have

$$
\begin{aligned}
& \lim _{x \longrightarrow x_{0}^{-}} F(x)=\lim _{x_{n} \uparrow x_{0}} \hat{\mathbb{E}}\left(\varphi\left(\frac{\xi}{x_{n}}\right)\right)=\hat{\mathbb{E}}\left(\varphi\left(\frac{\xi}{x_{0}}\right)\right)=F\left(x_{0}\right) \text { from } 0 \leq \varphi\left(\frac{\xi}{x_{n}}\right) \downarrow \varphi\left(\frac{\xi}{x_{0}}\right), \\
& \lim _{x \longrightarrow x_{0}^{+}} F(x)=\lim _{x_{n}^{\prime} \downarrow x_{0}} \hat{\mathbb{E}}\left(\varphi\left(\frac{\xi}{x_{n}^{\prime}}\right)\right)=\hat{\mathbb{E}}\left(\varphi\left(\frac{\xi}{x_{0}}\right)\right)=F\left(x_{0}\right) \text { from } 0 \leq \varphi\left(\frac{\xi}{x_{n}^{\prime}}\right) \uparrow \varphi\left(\frac{\xi}{x_{0}}\right), \\
& \lim _{x \longrightarrow 0^{+}} F(x)=\lim _{n \longrightarrow \infty} \hat{\mathbb{E}}(\varphi(n \xi))=1=F(0) \text { from } 0 \leq \varphi(n \xi) \uparrow 1 .
\end{aligned}
$$

Hence, $F(x)$ is continuous for $0 \leq x<\infty$. As well as $\lim _{x \rightarrow \infty} F(x)=\lim _{n \longrightarrow \infty} \widehat{\mathbb{E}}(\varphi(\xi / n))=0$ from $0 \leq \varphi(\xi / n) \downarrow 0$.

Therefore, let $\epsilon$ be an arbitrary positive number; there exist points $0<x_{1}<x_{2}, \ldots, x_{m}<\infty$ satisfying the conditions

$$
\begin{aligned}
1-F\left(x_{1}\right) & <\frac{\varepsilon}{2}, \\
F\left(x_{k}\right)-F\left(x_{k+1}\right) & <\frac{\varepsilon}{2}, \quad k=1, \ldots, m-1, \\
F\left(x_{m}\right) & <\frac{\varepsilon}{2} .
\end{aligned}
$$

Furthermore, by (19) and Remark 1, there exists a number $n_{0}$ such that for $n>n_{0}$ and we have

$$
\left|F_{n}\left(x_{k}\right)-F\left(x_{k}\right)\right|<\frac{\varepsilon}{2}, \quad k=1, \ldots, m .
$$

If $x_{k} \leq x<x_{k+1}(k=1, \ldots, m-1)$, then for $n>n_{0}$, we get $F_{n}(x)-F(x) \leq F_{n}\left(x_{k}\right)-F\left(x_{k}\right)+F\left(x_{k}\right)-F\left(x_{k+1}\right)<\varepsilon$, $F_{n}(x)-F(x) \geq F_{n}\left(x_{k+1}\right)-F\left(x_{k+1}\right)+F\left(x_{k+1}\right)-F\left(x_{k}\right)>-\varepsilon$.

If $0<x<x_{1}$, then for $n>n_{0}$,

$$
\begin{aligned}
& F_{n}(x)-F(x) \leq 1-F\left(x_{1}\right)<\varepsilon, \\
& F_{n}(x)-F(x) \geq F_{n}\left(x_{1}\right)-1=F_{n}\left(x_{1}\right)-F\left(x_{1}\right)+F\left(x_{1}\right)-1>-\varepsilon .
\end{aligned}
$$

If $x \geq x_{m}$, then for $n>n_{0}$,

$$
\begin{aligned}
& F_{n}(x)-F(x) \leq F_{n}\left(x_{m}\right)=F_{n}\left(x_{m}\right)-F\left(x_{m}\right)+F\left(x_{m}\right)<\varepsilon, \\
& F_{n}(x)-F(x) \geq 0-F\left(x_{m}\right)>-\varepsilon .
\end{aligned}
$$
holds.

Thus, $\left|F_{n}(x)-F(x)\right|<\varepsilon$ for all $x$ and $n>n_{0}$. That is, (31)

Next, we prove that (32).

Because $F$ is continuous on $[0, \infty), F$ is uniformly continuous on $[0,2]$. Therefore, for any $\epsilon>0$, there is $\delta>0$ (can be assumed $\delta<2$ ), such that $\forall x_{1}, x_{2} \in[0,2]$; if $\left|x_{1}-x_{2}\right|<\delta$, then

$$
\left|F\left(x_{1}\right)-F\left(x_{2}\right)\right|<\varepsilon .
$$

Let $\max (1 / 2,1-\delta / 2, \underline{\sigma} / \bar{\sigma})<\mu<1$, for any $x \in(0,1]$; we have $0<x / \mu \leq 2 x \leq 2$ and $|x / \mu-x|<\delta$. Hence,

$$
\Delta_{2}(x)=\widehat{\mathbb{E}}\left(\varphi\left(\frac{\xi}{x}\right)\right)-\widehat{\mathbb{E}}\left(\varphi\left(\frac{\mu \xi}{x}\right)\right)=F(x)-F\left(\frac{x}{\mu}\right)<\varepsilon .
$$

Thereby,

$$
\sup _{0<x \leq 1} \Delta_{2}(x) \leq \varepsilon
$$

For $x>1$, let $\Theta=\left\{\theta ; \theta_{t}\right.$ is $\mathscr{F}_{t}$-adapted process such that $\left.\underline{\sigma} / x \leq \theta_{t} \leq \bar{\sigma} / x\right\}$ and $\Theta^{\mu}=\left\{\theta ; \theta_{t} i\right.$ is $\mathscr{F}_{t}-$ adapted process such that that $\left.\underline{\sigma} / x \leq \theta_{t} \leq \mu \bar{\sigma} / x\right\}$; combining any $a>0, \xi / a \sim \mathcal{N}(0$, $\left.\left[\underline{\sigma}^{2} / a^{2}, \bar{\sigma}^{2} / a^{2}\right]\right)$ under $\widehat{\mathbb{E}}$. By Lemma 4 ,

$$
\begin{aligned}
\Delta_{2}(x) & =\hat{\mathbb{E}}\left(\varphi\left(\frac{\xi}{x}\right)\right)-\hat{\mathbb{E}}\left(\varphi\left(\frac{\mu \xi}{x}\right)\right) \\
& \leq \sup _{\theta \in \Theta} E_{P}\left(\varphi\left(\int_{0}^{1} \theta_{s} \mathrm{~d} B_{s}\right)\right)-\sup _{\theta \in \Theta^{\mu}} E_{P}\left(\varphi\left(\int_{0}^{1} \theta_{s} \mathrm{~d} B_{s}\right)\right) \\
& \leq \sup _{\theta \in \Theta} E_{P}\left(\varphi\left(\int_{0}^{1} \theta_{s} \mathrm{~d} B_{s}\right)\right)-\sup _{\theta \in \Theta} E_{P}\left(\varphi\left(\int_{0}^{1}\left(\theta_{s} \wedge \frac{\mu \bar{\sigma}}{x}\right) \mathrm{d} B_{s}\right)\right) \\
& \leq \sup _{\theta \in \Theta} E_{P}\left(\varphi\left(\int_{0}^{1} \theta_{s} \mathrm{~d} B_{s}\right)-\varphi\left(\int_{0}^{1}\left(\theta_{s} \wedge \frac{\mu \bar{\sigma}}{x}\right) \mathrm{d} B_{s}\right)\right) \\
& \leq c_{\varphi} \sup _{\theta \in \Theta} E_{P}\left(\int_{0}^{1} \theta_{s} \mathrm{~d} B_{s}-\int_{0}^{1}\left(\theta_{s} \wedge \frac{\mu \bar{\sigma}}{x}\right) \mathrm{d} B_{s}\right) \\
& \leq c_{\varphi} \sup _{\theta \in \Theta} \sqrt{E_{P}\left(\int_{0}^{1}\left(\theta_{s}-\left(\theta_{s} \wedge \frac{\mu \bar{\sigma}}{x}\right)\right) \mathrm{d} B_{s}\right)^{2}} \\
& =c_{\varphi} \sup _{\theta \in \Theta} \sqrt{\int_{0}^{1} E_{P}\left(\theta_{s}-\left(\theta_{s} \wedge \frac{\mu \bar{\sigma}}{x}\right)\right)^{2} \mathrm{~d} s} \\
& \leq c_{\varphi} \frac{\bar{\sigma}(1-\mu)}{x},
\end{aligned}
$$

where $c_{\varphi}$ is the Lipschitz constant of $\varphi$.

Therefore, 


$$
\sup _{x>1} \Delta_{2}(x) \leq c_{\varphi} \bar{\sigma}(1-\mu) \longrightarrow 0, \quad \mu \longrightarrow 1^{-} .
$$

The combination of (41) and (32) is established. This completes the proof of Lemma 5.

\section{Main Results and Proofs}

Our results are stated as follows.

Theorem 1. Let $\left\{X, X_{n} ; n \geq 1\right\}$ be a sequence of independent and identically distributed random variables in $(\Omega, \mathscr{H}, \widehat{\mathbb{E}})$. We assume that $\widehat{\mathbb{E}}$ is continuous and

$$
\begin{aligned}
\hat{\mathbb{E}}(X) & =\hat{\mathbb{E}}(-X)=0, \\
\lim _{c \longrightarrow \infty} \hat{\mathbb{E}}\left(X_{1}^{2}-c\right)^{+} & =0, \\
\hat{\mathbb{E}}\left(X^{2}\right) & =\bar{\sigma}^{2}<\infty, \\
\hat{\varepsilon}\left(X^{2}\right) & =\underline{\sigma}^{2}, \\
C_{\mathbb{V}}\left(X^{2}\right) & <\infty .
\end{aligned}
$$

Then,

$$
\lim _{\varepsilon \longrightarrow 0} \varepsilon^{2} \sum_{n=1}^{\infty} \mathbb{V}\left(\left|S_{n}\right| \geq \varepsilon n\right)=C_{\mathbb{V}}\left(\xi^{2}\right),
$$

here and later, $\xi \sim \mathcal{N}\left(0,\left[\underline{\sigma}^{2}, \bar{\sigma}^{2}\right]\right)$ under $\widehat{\mathbb{E}}$.

Theorem 2. Under the conditions of Theorem 1, for $0 \leq p<2$,

$$
\lim _{\varepsilon \longrightarrow 0} \varepsilon^{2-p} \sum_{n=1}^{\infty} \frac{1}{n^{p}} C_{\mathbb{V}}\left(\left|S_{n}\right|^{p} I\left(\left|S_{n}\right| \geq \varepsilon n\right)=\frac{2}{2-p} C_{\mathbb{V}}\left(\xi^{2}\right) .\right.
$$

For $p=2$, we have the following theorem.

Theorem 3. Let $\left\{X, X_{n} ; n \geq 1\right\}$ be a sequence of independent and identically distributed random variables in $(\Omega, \mathscr{H}, \widehat{\mathbb{E}})$. We assume that $\widehat{\mathbb{E}}$ is continuous and

$$
\begin{aligned}
\hat{\mathbb{E}}(X)=\hat{\mathbb{E}}(-X) & =0, \\
\lim _{c \longrightarrow \infty} \hat{\mathbb{E}}\left(X_{1}^{2}-c\right)^{+} & =0, \\
\hat{\mathbb{E}}\left(X^{2}\right) & =\bar{\sigma}^{2}<\infty, \\
\hat{\varepsilon}\left(X^{2}\right) & =\underline{\sigma}^{2}, \\
C_{\mathbb{V}}\left(X^{2} \ln |X|\right) & <\infty .
\end{aligned}
$$

Then,

$$
\lim _{\varepsilon \longrightarrow 0} \frac{1}{\ln \varepsilon^{-1}} \sum_{n=1}^{\infty} \frac{1}{n^{2}} C_{\mathbb{V}}\left(\left|S_{n}\right|^{2} I\left(\left|S_{n}\right| \geq \varepsilon n\right)=2 C_{\mathbb{V}}\left(\xi^{2}\right) .\right.
$$

Remark 3. Theorems 1-3 extend the corresponding results obtained by Liu and Lin [20] from the probability space to sublinear expectation space.

Proof of Theorem 1. Note that

$$
\varepsilon^{2} \sum_{n=1}^{\infty} \mathbb{V}\left(\left|S_{n}\right| \geq \varepsilon n\right)=\varepsilon^{2} \sum_{n=1}^{\infty} \mathbb{V}(|\xi| \geq \varepsilon \sqrt{n})+\varepsilon^{2} \sum_{n=1}^{\infty}\left[\mathbb{V}\left(\left|S_{n}\right| \geq \varepsilon n\right)-\mathbb{V}(|\xi| \geq \varepsilon \sqrt{n})\right]:=I_{1}(\varepsilon)+I_{2}(\varepsilon)
$$

Hence, in order to establish (45), it suffices to prove that

Obviously, (50) follows from

$$
\begin{aligned}
& \lim _{\varepsilon \longrightarrow 0} I_{1}(\varepsilon)=C_{\mathbb{V}}\left(\xi^{2}\right), \\
& \lim _{\varepsilon \longrightarrow 0} I_{2}(\varepsilon)=0 .
\end{aligned}
$$

$$
\begin{aligned}
\lim _{\varepsilon \longrightarrow 0} I_{1}(\varepsilon) & =\lim _{\varepsilon \longrightarrow 0} \varepsilon^{2} \int_{1}^{\infty} \mathbb{V}(|\xi| \geq \varepsilon \sqrt{x}) \mathrm{d} x=\lim _{\varepsilon \longrightarrow 0} \int_{\mathcal{\varepsilon}^{2}}^{\infty} \mathbb{V}\left(|\xi|^{2} \geq y\right) \mathrm{d} y \\
& =\int_{0}^{\infty} \mathbb{V}\left(|\xi|^{2} \geq y\right) \mathrm{d} y=C_{\mathbb{V}}\left(\xi^{2}\right) .
\end{aligned}
$$

Without loss of generality, here and later, we assume that $\widehat{\mathbb{E}} X^{2}=1$. Let $M>12$,

$$
\left|I_{2}(\varepsilon)\right| \leq \varepsilon^{2} \sum_{n \leq\left[M \varepsilon^{-2}\right]}\left|\mathbb{V}\left(\left|S_{n}\right| \geq \varepsilon n\right)-\mathbb{V}(|\xi| \geq \varepsilon \sqrt{n})\right|+\varepsilon^{2} \sum_{n>\left[M \varepsilon^{-2}\right]} \mathbb{V}\left(\left|S_{n}\right| \geq \varepsilon n\right)+\varepsilon^{2} \sum_{n>\left[M \varepsilon^{-2}\right]} \mathbb{V}(|\xi| \geq \varepsilon \sqrt{n}):=I_{21}(\varepsilon)+I_{22}(\varepsilon)+I_{23}(\varepsilon) .
$$


Let $\triangle_{n}=\sup _{x \geq 0}\left|\mathbb{V}\left(\left|S_{n}\right| \geq \sqrt{n} x\right)-\mathbb{V}(|\xi| \geq x)\right|$, from (26), $\triangle_{n} \longrightarrow 0$ as $n \longrightarrow \infty$. So, by Toeplitz's lemma, if $x_{n} \longrightarrow x, \omega_{i} \geq 0, \quad$ and $\quad \sum_{i=1}^{n} \omega_{i} \longrightarrow \infty$, then $\left(\sum_{i=1}^{n} \omega_{i} x_{i} / \sum_{i=1}^{n} \omega_{i}\right) \longrightarrow x$,

$$
\begin{array}{r}
\lim _{\varepsilon \longrightarrow 0} I_{21}(\varepsilon) \leq M, \\
\lim _{\varepsilon \longrightarrow 0} \frac{\sum_{n \leq\left[M \varepsilon^{-2}\right]} \triangle_{n}}{M \varepsilon^{-2}}=0 .
\end{array}
$$

Taking $\varphi$ as the proof process of Lemma 5, by (7), (27) and identically distributed of $X, X_{i}$, for any $x>0$,

$$
\mathbb{V}\left(\left|X_{i}\right| \geq x\right) \leq \hat{\mathbb{E}}\left[\varphi\left(\frac{X_{i}}{x}\right)\right]=\hat{\mathbb{E}}\left[\varphi\left(\frac{X}{x}\right)\right] \leq \mathbb{V}(|X| \geq \mu x) .
$$

Hence, for $n>M \varepsilon^{-2}>12 \varepsilon^{-2}$, taking $x=\varepsilon n$ and $y=\varepsilon n / 12$ in Lemma $1(\mathrm{i})$,

$$
\mathbb{V}\left(S_{n} \geq \varepsilon n\right) \leq \sum_{i=1}^{n} \mathbb{V}\left(\left|X_{i}\right| \geq \frac{\varepsilon n}{12}\right)+\exp \left(-\frac{\varepsilon^{2} n^{2}}{2\left(\varepsilon^{2} n^{2} / 12+n\right)}\left\{1+\frac{2}{3} \ln \left(1+\frac{\varepsilon^{2} n}{12}\right)\right\}\right) \leq n \mathbb{V}\left(|X| \geq\left(\frac{\mu \varepsilon n}{12}\right)\right)+\frac{c}{\mathcal{E}^{4} n^{2}}
$$

from $\quad\left(\varepsilon^{2} n^{2} / 2\left(\varepsilon^{2} n^{2} / \quad 12+n\right)\right)\left\{1+(2 / 3) \ln \left(1+\left(\varepsilon^{2} n /\right.\right.\right.$ 12)) $\} \geq 2 \ln \left(1+\left(\varepsilon^{2} n / 12\right)\right)$.

Since $\left\{-X,-X_{i}\right\}$ also satisfies the conditions of Theorem 1 , we replace the $\left\{X, X_{i}\right\}$ with the $\left\{-X,-X_{i}\right\}$ in the upper form:

$$
\mathbb{V}\left(-S_{n} \geq \varepsilon n\right) \ll n \mathbb{V}\left(|X| \geq \frac{\mu \varepsilon n}{12}\right)+\frac{1}{\mathcal{E}^{4} n^{2}} .
$$

Therefore,

$$
\begin{aligned}
\mathbb{V}\left(\left|S_{n}\right| \geq \varepsilon n\right) \leq & \mathbb{V}\left(S_{n} \geq \varepsilon n\right)+\mathbb{V}\left(-S_{n} \geq \varepsilon n\right) \ll n \mathbb{V}\left(|X| \geq \frac{\mu \varepsilon n}{12}\right) \\
& +\frac{1}{\mathcal{E}^{4} n^{2}}
\end{aligned}
$$

$$
\begin{aligned}
& \text { More generally, for any } x>0 \text { and } n>M \varepsilon^{-2} \text {, we have } \\
& \mathbb{V}\left(\left|S_{n}\right| \geq(\varepsilon+x) n\right) \ll n \mathbb{V}\left(|X| \geq \frac{\mu(\varepsilon+x) n}{12}\right)+\frac{1}{(\varepsilon+x)^{4} n^{2}}
\end{aligned}
$$

This implies from Markov's inequality and (24) that

$I_{22}(\varepsilon)+I_{23}(\varepsilon) \ll \varepsilon^{2} \sum_{n>\left[M \varepsilon^{-2}\right]}\left(n \mathbb{V}\left(|X| \geq \frac{\mu \varepsilon n}{12}\right)+\frac{1}{\mathcal{E}^{4} n^{2}}+\frac{\widehat{\mathbb{E}}|\xi|^{4}}{\mathcal{\varepsilon}^{4} n^{2}}\right) \ll \varepsilon^{2} \int_{\varepsilon^{-2}}^{\infty} x \mathbb{V}(|X| \geq c \mathcal{E} x) \mathrm{d} x+M^{-1}(\operatorname{let} c \mathcal{E} x=y) \sim c \int_{c \varepsilon^{-1}}^{\infty} y \mathbb{V}(|X| \geq y) \mathrm{d} y+M^{-1}$

Let $\varepsilon \longrightarrow 0$ first; then, let $M \longrightarrow \infty$; we get

$$
\lim _{\varepsilon \longrightarrow 0}\left(I_{22}(\varepsilon)+I_{23}(\varepsilon)\right)=0,
$$

from (44) and (15).

From this, combining with (53) and (54), (51) is established. This completes the proof of Theorem 1 .
Proof of Theorem 2. Since $C_{\mathbb{V}}\left(\left|S_{n}\right|^{p} I\left(\left|S_{n}\right| \geq \varepsilon n\right)\right)=\mathbb{V}\left(\left|S_{n}\right| \geq \varepsilon n\right)$ when $p=0$, so by Theorem 1 , we only discuss the case $0<p<2$. Note that

$$
\varepsilon^{2-p} \sum_{n=1}^{\infty} \frac{1}{n^{p}} C_{\mathbb{V}}\left(\left|S_{n}\right|^{p} I\left(\left|S_{n}\right| \geq \varepsilon n\right)\right)=\varepsilon^{2} \sum_{n=1}^{\infty} \mathbb{V}\left(\left|S_{n}\right| \geq \varepsilon n\right)+\varepsilon^{2-p} \sum_{n=1}^{\infty} \frac{1}{n^{p}} \int_{\varepsilon n}^{\infty} p x^{p-1} \mathbb{V}\left(\left|S_{n}\right| \geq x\right) \mathrm{d} x
$$

Hence, from Theorem 1, in order to establish (46), it suffices to prove that

$$
\lim _{\varepsilon \longrightarrow 0} \varepsilon^{2-p} \sum_{n=1}^{\infty} \frac{1}{n^{p}} \int_{\varepsilon n}^{\infty} p x^{p-1} \mathbb{V}\left(\left|S_{n}\right| \geq x\right) \mathrm{d} x=\frac{p}{2-p} C_{\mathbb{V}}\left(\xi^{2}\right)
$$


Let $M \geq 12$. Note that

$$
\begin{aligned}
\varepsilon^{2-} p & \sum_{n=1}^{\infty} \frac{1}{n^{p}} \int_{\varepsilon n}^{\infty} p x^{p-1} \mathbb{V}\left(\left|S_{n}\right| \geq x\right) \mathrm{d} x \\
= & \varepsilon^{2-p} \sum_{n=1}^{\infty} \frac{1}{n^{p}} \int_{\varepsilon n}^{\infty} p x^{p-1} \mathbb{V}\left(|\xi| \geq \frac{x}{\sqrt{n}}\right) \mathrm{d} x \\
& +\varepsilon^{2-p} \sum_{n \leq\left[M \varepsilon^{-2}\right]} \frac{1}{n^{p}} \int_{\varepsilon n}^{\infty} p x^{p-1}\left(\mathbb{V}\left(\left|S_{n}\right| \geq x\right)-\mathbb{V}\left(|\xi| \geq \frac{x}{\sqrt{n}}\right)\right) \mathrm{d} x \\
& +\varepsilon^{2-p} \sum_{n>\left[M \varepsilon^{-2}\right]} \frac{1}{n^{p}} \int_{\varepsilon n}^{\infty} p x^{p-1}\left(\mathbb{V}\left(\left|S_{n}\right| \geq x\right)-\mathbb{V}\left(|\xi| \geq \frac{x}{\sqrt{n}}\right)\right) \mathrm{d} x \\
:= & J_{1}(\varepsilon)+J_{2}(\varepsilon)+J_{3}(\varepsilon) .
\end{aligned}
$$

Hence, in order to establish (63), it suffices to prove that

$$
\begin{aligned}
\lim _{\varepsilon \longrightarrow 0} J_{1}(\varepsilon) & =\frac{p}{2-p} C_{\mathbb{V}}\left(\xi^{2}\right), \\
\lim _{\varepsilon \longrightarrow 0} J_{2}(\varepsilon) & =0, \\
\lim _{M \longrightarrow \infty} \limsup _{\varepsilon \longrightarrow 0}\left|J_{3}(\varepsilon)\right| & =0 .
\end{aligned}
$$

We first prove (65); let $y:=x / \sqrt{n}$, then

$$
\begin{aligned}
\lim _{\varepsilon \longrightarrow 0} J_{1}(\varepsilon)= & \lim _{\varepsilon \longrightarrow 0} \varepsilon^{2-p} \sum_{n=1}^{\infty} \frac{1}{n^{p / 2}} \int_{\varepsilon \sqrt{n}}^{\infty} p y^{p-1} \mathbb{V}(|\xi| \geq y) \mathrm{d} y \\
= & \lim _{\varepsilon \longrightarrow 0} \varepsilon^{2-p} \int_{1}^{\infty} \frac{1}{t^{p / 2}} \mathrm{~d} \int_{\varepsilon \sqrt{t}}^{\infty} p y^{p-1} \mathbb{V}(|\xi| \geq y) \mathrm{d} y \\
= & \lim _{\varepsilon \longrightarrow 0} \varepsilon^{2-p} \int_{\varepsilon}^{\infty} p y^{p-1} \mathbb{V}(|\xi| \geq y) \mathrm{d} y \int_{1}^{y^{2} / \varepsilon^{2}} \frac{1}{t^{p / 2}} \mathrm{~d} t \\
= & \lim _{\varepsilon \longrightarrow 0} \frac{2 p}{2-p} \varepsilon^{2-p} \int_{\varepsilon}^{\infty} y^{p-1} \mathbb{V}(|\xi| \geq y)\left(\left(\frac{y^{2}}{\varepsilon^{2}}\right)^{-p / 2+1}-1\right) \mathrm{d} y \\
= & \frac{2 p}{2-p} \int_{0}^{\infty} y \mathbb{V}(|\xi| \geq y) \mathrm{d} y \\
& -\lim _{\varepsilon \longrightarrow 0} \frac{2 p}{2-p} \varepsilon^{2-p} \int_{\varepsilon}^{\infty} y^{p-1} \mathbb{V}(|\xi| \geq y) \mathrm{d} y \\
= & \frac{p}{2-p} C_{\mathbb{V}}\left(\xi^{2}\right),
\end{aligned}
$$

from (25).

Now, we prove (66). Let $b_{n}=\left(\sqrt{n} \triangle_{n}^{1 /(2 p)}\right)^{-1}$. Then,

$$
\begin{aligned}
\left|J_{2}(\varepsilon)\right| \leq \varepsilon^{2-p} \sum_{n \leq\left[M \varepsilon^{-2}\right]} \frac{1}{n^{p}} \int_{\varepsilon n}^{\infty} p x^{p-1}\left|\mathbb{V}\left(\left|S_{n}\right| \geq x\right)-\mathbb{V}\left(|\xi| \geq \frac{x}{\sqrt{n}}\right)\right| \mathrm{d} x \\
\leq \varepsilon^{2-p} \sum_{n \leq\left[M \varepsilon^{-2}\right]} \int_{0}^{\infty} p(y+\varepsilon)^{p-1} \mid \mathbb{V}\left(\left|S_{n}\right| \geq(y+\varepsilon) n\right) \\
\quad-\mathbb{V}(|\xi| \geq(y+\varepsilon) \sqrt{n}) \mid \mathrm{d} y \\
\leq \varepsilon^{2-p} \sum_{n \leq\left[M \varepsilon^{-2}\right]} \int_{0}^{b_{n}} p(y+\varepsilon)^{p-1} \triangle_{n} \mathrm{~d} y \\
\quad+\varepsilon^{2-p} \sum_{n \leq\left[M \varepsilon^{-2}\right]} \int_{b_{n}}^{\infty} p(y+\varepsilon)^{p-1}\left(\mathbb{V}\left(\left|S_{n}\right| \geq(y+\varepsilon) n\right)\right. \\
\quad+\mathbb{V}(|\xi| \geq(y+\varepsilon) \sqrt{n})) \mathrm{d} y \\
:=\varepsilon^{2-p} \sum_{n \leq\left[M \varepsilon^{-2}\right]} \frac{1}{n^{p / 2}}\left(J_{21}(\varepsilon)+J_{22}(\varepsilon)\right),
\end{aligned}
$$

where

$$
\begin{aligned}
J_{21}(\varepsilon) & =n^{p / 2} \int_{0}^{b_{n}} p(y+\varepsilon)^{p-1} \triangle_{n} \mathrm{~d} y \\
J_{22}(\varepsilon) & =n^{p / 2} \int_{b_{n}}^{\infty} p(y+\varepsilon)^{p-1}\left(\mathbb{V}\left(\left|S_{n}\right| \geq(y+\varepsilon) n\right)+\mathbb{V}(|\xi|\right. \\
& \geq(y+\varepsilon) \sqrt{n})) \mathrm{d} y .
\end{aligned}
$$

Since $n \leq M \varepsilon^{-2}$ implies $\varepsilon \sqrt{n} \leq \sqrt{M}$, one can easily obtain that

$$
J_{21}(\varepsilon) \leq \Delta_{n} n^{p / 2}\left(\frac{1}{\sqrt{n} \triangle_{n}^{1 /(2 p)}}+\varepsilon\right)^{p} \leq\left(\Delta_{n}^{1 / 2 p}+\sqrt{M} \Delta_{n}^{1 / p}\right)^{p}
$$

$$
\ll \Delta_{n}^{1 / 2} \longrightarrow 0 \text { as } n \longrightarrow \infty \text {. }
$$


By Lemma 1 (ii), Markov's inequality, and (24), we get

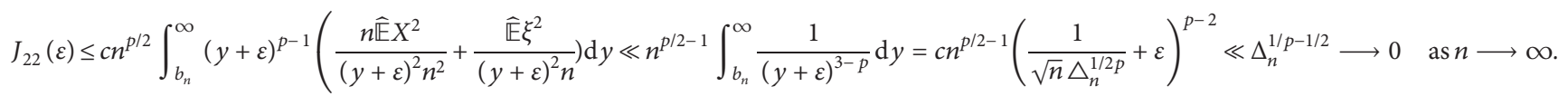

From (69)-(72) and That is, (66) is established.

$\sum_{n \leq\left[M \varepsilon^{-2}\right]}\left(1 / n^{p / 2}\right)=O\left(\varepsilon^{p-2}\right) \longrightarrow \infty, \varepsilon \longrightarrow 0, \quad$ using $\quad$ Toe- $\quad$ Finally, we prove (67):

plitz's lemma, we get

$$
\begin{aligned}
\left|J_{2}(\varepsilon)\right| & \leq \varepsilon^{2-p} \sum_{n \leq\left[M \varepsilon^{-2}\right]} \frac{J_{21}(\varepsilon)+J_{22}(\varepsilon)}{n^{p / 2}} \\
& \ll \frac{\sum_{n \leq\left[M \varepsilon^{-2}\right]}\left(\triangle_{n}^{1 / 2}+\triangle_{n}^{1 / p-1 / 2} / n^{p / 2}\right)}{\sum_{n \leq\left[M \varepsilon^{-2}\right]}\left(1 / n^{p / 2}\right)} \longrightarrow 0, \quad \varepsilon \longrightarrow 0 .
\end{aligned}
$$

$$
\begin{aligned}
\left|J_{3}(\varepsilon)\right| & \leq \varepsilon^{2-p} \sum_{n>\left[M \varepsilon^{-2}\right]} \frac{1}{n^{p}} \int_{\varepsilon n}^{\infty} p x^{p-1}\left(\mathbb{V}\left(\left|S_{n}\right| \geq x\right)+\mathbb{V}\left(|\xi| \geq \frac{x}{\sqrt{n}}\right)\right) \mathrm{d} x\left(\text { let } y=\frac{x}{n-\varepsilon}\right) \\
& \leq \varepsilon^{2-p} \sum_{n>\left[M \varepsilon^{-2}\right]} \int_{0}^{\infty} p(y+\varepsilon)^{p-1}\left(\mathbb{V}\left(\left|S_{n}\right| \geq(y+\varepsilon) n\right)+\mathbb{V}(|\xi| \geq(y+\varepsilon) \sqrt{n})\right) \mathrm{d} y .
\end{aligned}
$$

By (59), Markov's inequality, and (24),

$$
\begin{aligned}
\left|J_{3}(\varepsilon)\right| & \ll \varepsilon^{2-p} \sum_{n>\left[M \varepsilon^{-2}\right]} \int_{0}^{\infty}(y+\varepsilon)^{p-1}\left(n \mathbb{V}(|X| \geq c(y+\varepsilon) n)+\frac{1}{(y+\varepsilon)^{4} n^{2}}+\frac{\hat{\mathbb{E}}|\xi|^{4}}{(y+\varepsilon)^{4} n^{2}}\right) \mathrm{d} y \\
& \ll \varepsilon^{2-p} \int_{M \varepsilon^{-2}}^{\infty} x \mathbb{V}(|X| \geq c(y+\varepsilon) x) \mathrm{d} x \int_{0}^{\infty}(y+\varepsilon)^{p-1} d y+\varepsilon^{2-p} \sum_{n>\left[M \varepsilon^{-2}\right]} \frac{1}{n^{2}} \int_{0}^{\infty}(y+\varepsilon)^{p-5} \mathrm{~d} y \\
& \ll \varepsilon^{2-p} \int_{0}^{\infty}(y+\varepsilon)^{p-1} d y \int_{c M \varepsilon^{-2}(y+\varepsilon)}^{\infty} \frac{t}{(y+\varepsilon)^{2}} \mathbb{V}(|X| \geq t) \mathrm{d} t+M^{-1}(\operatorname{let} c(y+\varepsilon) x=t) \\
& \ll \varepsilon^{2-p} \int_{0}^{\infty}(y+\varepsilon)^{p-3} \mathrm{~d} y \int_{c \varepsilon^{-1}}^{\infty} t \mathbb{V}(|X| \geq t) \mathrm{d} t+M^{-1} \\
& =\int_{c \varepsilon^{-1}}^{\infty} t \mathbb{V}(|X| \geq t) \mathrm{d} t+M^{-1} .
\end{aligned}
$$

Let $\varepsilon \longrightarrow 0$ first, then let $M \longrightarrow \infty$, we get (67) from

Proof of Theorem 3. Note that

(44) and (15). This completes the proof of Theorem 2.

$$
\sum_{n=1}^{\infty} \frac{1}{n^{2}} C_{\mathbb{V}}\left(S_{n}^{2} I\left(\left|S_{n}\right| \geq \varepsilon n\right)\right)=\varepsilon^{2} \sum_{n=1}^{\infty} \mathbb{V}\left(\left|S_{n}\right| \geq \varepsilon n\right)+\sum_{n=1}^{\infty} \frac{1}{n^{2}} \int_{\varepsilon n}^{\infty} 2 x \mathbb{V}\left(\left|S_{n}\right| \geq x\right) \mathrm{d} x
$$


Hence, by Theorem 1, in order to establish (48), it suffices to prove that

$$
\lim _{\varepsilon \longrightarrow 0} \frac{1}{\ln \varepsilon^{-1}} \sum_{n=1}^{\infty} \frac{1}{n^{2}} \int_{\varepsilon n}^{\infty} 2 x \mathbb{V}\left(\left|S_{n}\right| \geq x\right) \mathrm{d} x=2 C_{\mathbb{V}}\left(\xi^{2}\right) .
$$

However,

$$
\begin{aligned}
& \frac{1}{\ln \varepsilon^{-1}} \sum_{n=1}^{\infty} \frac{1}{n^{2}} \int_{\varepsilon n}^{\infty} 2 x \mathbb{V}\left(\left|S_{n}\right| \geq x\right) \mathrm{d} x \\
& =\frac{1}{\ln \varepsilon^{-1}} \sum_{n=1}^{\infty} \frac{1}{n^{2}} \int_{\varepsilon n}^{\infty} 2 x \mathbb{V}\left(|\xi| \geq \frac{x}{\sqrt{n}}\right) \mathrm{d} x \\
& \quad+\frac{1}{\ln \varepsilon^{-1}} \sum_{n \leq M \varepsilon^{-2}} \frac{1}{n^{2}} \int_{\varepsilon n}^{\infty} 2 x\left(\mathbb{V}\left(\left|S_{n}\right| \geq x\right)-\mathbb{V}\left(|\xi| \geq \frac{x}{\sqrt{n}}\right)\right) \mathrm{d} x \\
& \quad+\frac{1}{\ln \varepsilon^{-1}} \sum_{n>M \varepsilon^{-2}} \frac{1}{n^{2}} \int_{\varepsilon n}^{\infty} 2 x\left(\mathbb{V}\left(\left|S_{n}\right| \geq x\right)-\mathbb{V}\left(|\xi| \geq \frac{x}{\sqrt{n}}\right)\right) \mathrm{d} x \\
& \quad:=K_{1}(\varepsilon)+K_{2}(\varepsilon)+K_{3}(\varepsilon) .
\end{aligned}
$$

Hence, in order to establish (77), it suffices to prove that

$$
\begin{aligned}
& \lim _{\varepsilon \longrightarrow 0} K_{1}(\varepsilon)=2 C_{\mathbb{V}}\left(\xi^{2}\right), \\
& \lim _{\varepsilon \longrightarrow 0} K_{2}(\varepsilon)=0,
\end{aligned}
$$

$$
\lim _{M \longrightarrow \infty} \limsup _{\varepsilon \longrightarrow 0}\left|K_{3}(\varepsilon)\right|=0 .
$$

We first prove (79). Because $\lim _{y \longrightarrow 0^{+}} y|\ln y|=0$, there is $c_{1}>0$, such that $y|\ln y| \leq c_{1}$ for any $y \in(0,1]$. Therefore, combining with Markov's inequality and (24),

$$
\left|\int_{\varepsilon}^{\infty} y \ln y \mathbb{V}(|\xi| \geq y) \mathrm{d} y\right| \leq \int_{0}^{1} y|\ln y| \mathrm{d} y+\int_{1}^{\infty} y \ln y \frac{\widehat{\mathbb{E}}|\xi|^{3}}{y^{3}} \mathrm{~d} y \leq c_{1}+\widehat{\mathbb{E}}|\xi|^{3} \int_{1}^{\infty} \frac{\ln y}{y^{2}} \mathrm{~d} y=c<\infty
$$

Thus, (79) follows

$$
\begin{aligned}
\lim _{\varepsilon \longrightarrow 0} K_{1}(\varepsilon)= & \lim _{\varepsilon \longrightarrow 0} \frac{1}{\ln \varepsilon^{-1}} \sum_{n=1}^{\infty} \frac{1}{n} \int_{\varepsilon \sqrt{n}}^{\infty} 2 y \mathbb{V}(|\xi| \geq y) \mathrm{d} y\left(\text { let } y=\frac{x}{\sqrt{n}}\right) \\
= & \lim _{\varepsilon \longrightarrow 0} \frac{1}{\ln \varepsilon^{-1}} \int_{1}^{\infty} \frac{1}{t} \mathrm{~d} t \int_{\varepsilon \sqrt{t}}^{\infty} 2 y \mathbb{V}(|\xi| \geq y) \mathrm{d} y(\text { let } u=\varepsilon \sqrt{t}) \\
= & \lim _{\varepsilon \longrightarrow 0} \frac{1}{\ln \varepsilon^{-1}} \int_{\varepsilon}^{\infty} \frac{2 d u}{u} \int_{u}^{\infty} 2 y \mathbb{V}(|\xi| \geq y) \mathrm{d} y \\
= & \lim _{\varepsilon \longrightarrow 0} \frac{1}{\ln \varepsilon^{-1}} \int_{\varepsilon}^{\infty} 4 y \mathbb{V}(|\xi| \geq y) \mathrm{d} y \int_{\varepsilon}^{y} \frac{1}{u} \mathrm{~d} u \\
= & \lim _{\varepsilon \longrightarrow 0} \frac{1}{\ln \varepsilon^{-1}} \int_{\varepsilon}^{\infty} 4 y \ln y \mathbb{V}(|\xi| \geq y) \mathrm{d} y \\
& +\int_{0}^{\infty} \frac{4 y \mathbb{V}(|\xi| \geq y) \mathrm{d} y}{=} \\
& 2 C_{\mathbb{V}}\left(\xi^{2}\right) .
\end{aligned}
$$


Now, we prove (80). Let $d_{n}=\left(\sqrt{n} \triangle_{n}^{1 / 4}\right)^{-1}$. Then,

$$
\begin{aligned}
\left|K_{2}(\varepsilon)\right| \leq & \frac{1}{\ln \varepsilon^{-1}} \sum_{n \leq\left[M \varepsilon^{-2}\right]} \frac{1}{n^{2}} \int_{\varepsilon n}^{\infty} 2 x\left|\mathbb{V}\left(\left|S_{n}\right| \geq x\right)-\mathbb{V}\left(|\xi| \geq \frac{x}{\sqrt{n}}\right)\right| \mathrm{d} x(\operatorname{let}(y+\varepsilon) n=x) \\
= & \frac{1}{\ln \varepsilon^{-1}} \sum_{n \leq\left[M \varepsilon^{-2}\right]} \int_{0}^{\infty} 2(y+\varepsilon)\left|\mathbb{V}\left(\left|S_{n}\right| \geq(y+\varepsilon) n\right)-\mathbb{V}(|\xi| \geq(y+\varepsilon) \sqrt{n})\right| \mathrm{d} y \\
\leq & \frac{1}{\ln \varepsilon^{-1}} \sum_{n \leq\left[M \varepsilon^{-2}\right]} \int_{0}^{d_{n}} 2(y+\varepsilon) \triangle_{n} \mathrm{~d} y \\
& +\frac{1}{\ln \varepsilon^{-1}} \sum_{n \leq\left[M \varepsilon^{-2}\right]} \int_{d_{n}}^{\infty} 2(y+\varepsilon)\left(\mathbb{V}\left(\left|S_{n}\right| \geq(y+\varepsilon) n\right)+\mathbb{V}(|\xi| \geq(y+\varepsilon) \sqrt{n})\right) \mathrm{d} y \\
:= & \frac{1}{\ln \varepsilon^{-1}} \sum_{n \leq\left[M \varepsilon^{-2}\right]} \frac{1}{n}\left(K_{21}(\varepsilon)+K_{22}(\varepsilon)\right),
\end{aligned}
$$

where

$$
\begin{aligned}
& K_{21}(\varepsilon)=n \int_{0}^{d_{n}} 2(y+\varepsilon) \triangle_{n} \mathrm{~d} y, \\
& K_{22}(\varepsilon)=n \int_{d_{n}}^{\infty} 2(y+\varepsilon)\left(\mathbb{V}\left(\left|S_{n}\right| \geq(y+\varepsilon) n\right)+\mathbb{V}(|\xi| \geq(y+\varepsilon) \sqrt{n})\right) \mathrm{d} y .
\end{aligned}
$$
that

Since $n \leq M \varepsilon^{-2}$ implies $\epsilon \sqrt{n} \leq \sqrt{M}$, one can easily obtain

$$
K_{21}(\varepsilon) \leq \Delta_{n} n\left(\frac{1}{\sqrt{n} \triangle_{n}^{1 / 4}}+\varepsilon\right)^{2} \leq\left(\Delta_{n}^{1 / 4}+\sqrt{M} \Delta_{n}^{1 / 2}\right)^{2} \ll \Delta_{n}^{1 / 2} \longrightarrow 0 \quad \text { as } n \longrightarrow \infty .
$$

By (59), Markov's inequality, (24), and $c n\left(d_{n}+\varepsilon\right) \geq c \sqrt{n}$,

we get

$$
\begin{aligned}
K_{22}(\varepsilon) & \leq c n \int_{d_{n}}^{\infty} 2(y+\varepsilon)\left(n \mathbb{V}(|X| \geq c n(y+\varepsilon))+\frac{1}{n^{2}(y+\varepsilon)^{4}}+\frac{\widehat{\mathbb{E}}|\xi|^{4}}{n^{2}(y+\varepsilon)^{4}}\right) \mathrm{d} y \\
& =c \int_{c n\left(d_{n}+\varepsilon\right)}^{\infty} t \mathbb{V}(|X| \geq t) \mathrm{d} t+c \int_{d_{n}}^{\infty} \frac{1}{n(y+\varepsilon)^{3}} \mathrm{~d} y \quad(\operatorname{let} c n(y+\varepsilon)=t) \\
& \ll \int_{c \sqrt{n}}^{\infty} t \mathbb{V}(|X| \geq t) \mathrm{d} t+\frac{1}{n}\left(\frac{1}{\sqrt{n} \triangle_{n}^{1 / 4}}+\varepsilon\right)^{-2} \\
& \longrightarrow 0 \text { as } n \longrightarrow \infty .
\end{aligned}
$$


From (84)-(87) and $\sum_{n \leq\left[M \varepsilon^{-2}\right]} 1 / n=O\left(\ln \varepsilon^{-1}\right) \longrightarrow \infty, \varepsilon \longrightarrow 0$, using Toeplitz's lemma, (80) follows

$$
\left|K_{2}(\varepsilon)\right| \leq \frac{1}{\ln \varepsilon^{-1}} \sum_{n \leq\left[M \varepsilon^{-2}\right]} \frac{\triangle_{n}^{1 / 2}+K_{22}(\varepsilon)}{n} \ll \frac{\sum_{n \leq\left[M \varepsilon^{-2}\right]}\left(\triangle_{n}^{1 / 2}+K_{22}(\varepsilon) / n\right)}{\sum_{n \leq\left[M \varepsilon^{-2}\right]}(1 / n)} \longrightarrow 0, \quad \text { as } \varepsilon \longrightarrow 0
$$

Finally, we prove (81) as follows:

$$
\begin{aligned}
\left|K_{3}(\varepsilon)\right| & \leq \frac{1}{\ln \varepsilon^{-1}} \sum_{n>\left[M \varepsilon^{-2}\right]} \frac{1}{n^{2}} \int_{\varepsilon n}^{\infty} 2 x\left(\mathbb{V}\left(\left|S_{n}\right| \geq x\right)+\mathbb{V}\left(|\xi| \geq \frac{x}{\sqrt{n}}\right)\right) \mathrm{d} x\left(\text { let } y=\frac{x}{n-\varepsilon}\right) \\
& =\frac{1}{\ln \varepsilon^{-1}} \sum_{n>\left[M \varepsilon^{-2}\right]} \int_{0}^{\infty} 2(y+\varepsilon)\left(\mathbb{V}\left(\left|S_{n}\right| \geq(y+\varepsilon) n\right)+\mathbb{V}(|\xi| \geq(y+\varepsilon) \sqrt{n})\right) \mathrm{d} y .
\end{aligned}
$$

By (59), Markov's inequality, and (24),

$$
\begin{aligned}
\left|K_{3}(\varepsilon)\right| & \ll \frac{1}{\ln \varepsilon^{-1}} \sum_{n>\left[M \varepsilon^{-2}\right]} \int_{0}^{\infty}(y+\varepsilon)\left(n \mathbb{V}(|X| \geq c(y+\varepsilon) n)+\frac{1}{(y+\varepsilon)^{4} n^{2}}+\frac{\widehat{\mathbb{E}}|\xi|^{4}}{(y+\varepsilon)^{4} n^{2}}\right) \mathrm{d} y \\
& \sim \frac{1}{\ln \varepsilon^{-1}} \int_{M \varepsilon^{-2}}^{\infty} \int_{0}^{\infty}(y+\varepsilon) x \mathbb{V}(|X| \geq c(y+\varepsilon) x) \mathrm{d} y \mathrm{~d} x+\frac{c}{\ln \varepsilon^{-1}} \sum_{n>\left[M \varepsilon^{-2}\right]} \frac{1}{n^{2}} \int_{0}^{\infty}(y+\varepsilon)^{-3} \mathrm{~d} y \\
& \left.\sim \frac{1}{\ln \varepsilon^{-1}} \int_{0}^{\infty}(y+\varepsilon) \int_{c M \varepsilon^{-2}(y+\varepsilon)}^{\infty} \frac{t}{(y+\varepsilon)^{2}} \mathbb{V}(|X| \geq t)\right) \mathrm{d} t \mathrm{~d} y+\frac{2 c M^{-1}}{\ln \varepsilon^{-1}} \\
& \ll \frac{1}{\ln \varepsilon^{-1}} \int_{c \varepsilon^{-1}}^{\infty} t \mathbb{V}(|X| \geq t) \mathrm{d} t \int_{0}^{\varepsilon^{2} t /(c M)-\varepsilon} \frac{1}{y+\varepsilon} \mathrm{d} y+M^{-1} \\
& =\frac{1}{\ln \varepsilon^{-1}} \int_{c \varepsilon^{-1}}^{\infty} t \mathbb{V}(|X| \geq t)\left(\ln \frac{\varepsilon^{2}}{c M}+\ln t+\ln \varepsilon^{-1}\right) \mathrm{d} t+M^{-1} \\
& \leq \frac{1}{\ln \varepsilon^{-1}} \int_{c \varepsilon^{-1}}^{\infty} t \ln \mathbb{V}(|X| \geq t) \mathrm{d} t+\int_{c \varepsilon^{-1}}^{\infty} t \mathbb{V}(|X| \geq t) \mathrm{d} t+M^{-1}
\end{aligned}
$$

Let $\varepsilon \longrightarrow 0$ first and then let $M \longrightarrow \infty$; we get (81) from (47) and (16). This completes the proof of Theorem 3.

\section{Data Availability}

No data were used to support this study.

\section{Conflicts of Interest}

The authors declare no conflicts of interest.

\section{Acknowledgments}

This research was supported by the National Natural Science Foundation of China (11661029) and the Support Program of the Guangxi China Science Foundation (2018GXNSFAA281011).

\section{References}

[1] S. Peng, "G-expectation, G-brownian motion and related stochastic calculus of itô type," Stochastic Analysis and Applications, vol. 2, no. 4, pp. 541-567, 2007.

[2] L. Denis and C. Martini, "A theoretical framework for the pricing of contingent claims in the presence of model uncertainty," The Annals of Applied Probability, vol. 16, no. 2, pp. 827-852, 2006.

[3] I. Gilboa, "Expected utility with purely subjective non-additive probabilities," Journal of Mathematical Economics, vol. 16, no. 1, pp. 65-8810, 1987. 
[4] M. Marinacci, "Limit laws for non-additive probabilities and their frequentist interpretation," Journal of Economic Theory, vol. 84, no. 2, pp. 145-195, 1999.

[5] S. G. Peng, "BSDE and related g-expectation," Pitman Research Notes in Mathematics Series, vol. 364, pp. 141-159, 1997.

[6] S. Peng, "Monotonic limit theorem of BSDE and nonlinear decomposition theorem of Doob-Meyers type," Probability Theory and Related Fields, vol. 113, no. 4, pp. 473-499, 1999.

[7] S. G. Peng, "Nonlinear expectations and stochastic calculus under uncertainty," 2010, https://arxiv.org/abs/1002.4546.

[8] S. Peng, "Survey on normal distributions, central limit theorem, Brownian motion and the related stochastic calculus under sublinear expectations," Science in China Series A: Mathematics, vol. 52, no. 7, pp. 1391-1411, 2009.

[9] L. X. Zhang, "Strong limit theorems for extended independent and extended negatively dependent random variables under non-linear expectations," 2016, https://arxiv.org/abs/1608. 00710.

[10] L. X. Zhang, "Exponential inequalities under the sub-linear expectations with applications to laws of the iterated logarithm," Science China Mathematics, vol. 59, no. 12, pp. 2503-2526, 2016.

[11] L. Zhang, "Rosenthal's inequalities for independent and negatively dependent random variables under sub-linear expectations with applications," Science China Mathematics, vol. 59 , no. 4 , pp. 751-768, 2016c.

[12] C. Hu, "A strong law of large numbers for sub-linear expectation under a general moment condition," Statistics and Probability Letters, vol. 119, pp. 248-258, 2016.

[13] Z. Chen, "Strong laws of large numbers for sub-linear expectations," Science China Mathematics, vol. 59, no. 5, pp. 945-954, 2016.

[14] Q. Wu and Y. Jiang, "Strong law of large numbers and Chover's law of the iterated logarithm under sub-linear expectations," Journal of Mathematical Analysis and Applications, vol. 460, no. 1, pp. 252-270, 2018.

[15] Y. Wu, X. Wang, and L. Zhang, "On the asymptotic approximation of inverse moment under sub-linear expectations," Journal of Mathematical Analysis and Applications, vol. 468, no. 1, pp. 182-196, 2018.

[16] M. Xi, Y. Wu, and X. Wang, "Complete convergence for arrays of rowwise END random variables and its statistical applications under sub-linear expectations," Journal of the Korean Statistical Society, vol. 48, no. 3, pp. 412-425, 2019.

[17] Y. Lin and X. Feng, "Complete convergence and strong law of large numbers for arrays of random variables under sublinear expectations," Communications in Statistics-Theory and Methods, pp. 1-17, 2019.

[18] P. L. Hsu and H. Robbins, "Complete convergence and the law of large numbers," Proceedings of the National Academy of Sciences, vol. 33, no. 2, pp. 25-31, 1947.

[19] C. C. Heyde, "A supplement to the strong law of large numbers," Journal of Applied Probability, vol. 12, no. 1, pp. 173-175, 1975.

[20] W. Liu and Z. Lin, "Precise asymptotics for a new kind of complete moment convergence," Statistics \& Probability Letters, vol. 76, no. 16, pp. 1787-1799, 2006.

[21] Y. Zhao, "Precise rates in complete moment convergence for $\rho$-mixing sequences," Journal of Mathematical Analysis and Applications, vol. 339, no. 1, pp. 553-565, 2008.

[22] J. Li, "Precise asymptotics of moving average process under $\varphi$-mixing assumption," Journal of the Korean Mathematical Society, vol. 49, no. 2, pp. 235-249, 2012.
[23] H. Zhou, "Precise asymptotics in the law of the logarithm for U-statistics," Acta Mathematica Scientia, Ser. A, vol. 32, no. 1, pp. 41-55, 2012.

[24] A. Gut and J. Steinebach, "Precise asymptotics-a general approach," Acta Mathematica Hungarica, vol. 138, no. 4, pp. 365-385, 2013.

[25] J.-J. He and T.-F. Xie, "Asymptotic property for some series of probability," Acta Mathematicae Applicatae Sinica, English Series, vol. 29, no. 1, pp. 179-186, 2013.

[26] X. Wang, X. Deng, L. Zheng, and S. Hu, "Complete convergence for arrays of rowwise negatively superadditive-dependent random variables and its applications," Statistics, vol. 48, no. 4, pp. 834-850, 2014.

[27] X. Wang, C. Xu, T.-C. Hu, A. Volodin, and S. Hu, "On complete convergence for widely orthant-dependent random variables and its applications in nonparametric regression models," TEST, vol. 23, no. 3, pp. 607-629, 2014.

[28] X. Wang, A. Shen, Z. Chen, and S. Hu, "Complete convergence for weighted sums of NSD random variables and its application in the EV regression model," TEST, vol. 24, no. 1, pp. 166-184, 2015.

[29] X. Wang, L. Zheng, C. Xu, and S. Hu, "Complete consistency for the estimator of nonparametric regression models based on extended negatively dependent errors," Statistics, vol. 49, no. 2, pp. 396-407, 2015.

[30] A. Spaataru, "Convergence and precise asymptotics for series involving self- normalized sums," Journal of Theoretical Probability, vol. 29, no. 1, pp. 267-276, 2016.

[31] L. Kong and H. Dai, "Convergence rate in precise asymptotics for Davis law of large numbers," Statistics \& Probability Letters, vol. 119, pp. 295-300, 2016.

[32] Z. J. Chen and F. Hu, "A law of the iterated logarithm under sublinear expectations," Journal of Financial Engineering, vol. 1, no. 2, pp. 131-153, 2014. 